\title{
Odontoma compuesto: Diagnóstico radiográfico y tratamiento quirúrgico de un caso clínico
}

\section{Compound odontoma: Radiographic diagnosis and surgical management of a clinical case}

Vázquez Diego J*, Gandini Pablo C**, Carbajal Eduardo E***

\section{RESUMEN}

Se presenta un caso clínico de odontoma compuesto asociado a un diente supernumerario y un canino retenido superior derecho. El paciente de 16 años de edad es de sexo masculino. Se realizan diversos estudios radiográficos para su diagnóstico, que consisten en técnica panorámica y técnicas intraorales.

Posteriormente se realizó la intervención quirúrgica para extirpar la lesión y las estructuras dentarias asociadas a ella. El estudio histopatológico confirmó el diagnóstico presuntivo. Basado en lo expuesto se analiza al odontoma compuesto según localización, sexo y edad habiendo realizado una revisión del tema según diversos autores.

Palabras clave: Odontoma compuesto, canino retenido, diente supernumerario, técnicas radiográficas, cirugía, caso clínico.

\section{SUMMARY}

A clinical case of a compound odontoma associated with a supernumerary tooth and an upper right impacted canine in a 16-year-old male, is reported. To perform the diagnosis, several radiographic studies were made, mainly consisting of panoramic and intraoral techniques. Then, a surgical procedure was carried out to remove both the lesion and the surrounding tooth structures. The histo-pathological examination confirmed our presumptive diagnosis. Therefore, based on these findings, the compound odontoma is analyzed according to its location, sex and age of patients as well, after reviewing other authors' opinions in the current literature.

Key words: Compound odontoma, impacted canine, supernumerary tooth, radiographic techniques, surgical procedures, clinical case.

Fecha de recepción: Febrero 2008.

Aceptado para publicación: Marzo 2008.

* Jefe de Trabajos Prácticos Regular. Cátedra de Radiología. Facultad de Odontología. Universidad de Buenos Aires. Argentina.

** Ayudante de Primera Regular. Cátedra de Cirugía B. M. F. III. Facultad de Odontología. Universidad de Buenos Aires. Argentina.

*** Profesor Adjunto Regular. Cátedra de Radiología. Facultad de Odontología. Universidad de Buenos Aires. Argentina.

Vázquez Diego J, Gandini Pablo C, Carbajal Eduardo E. Odontoma compuesto: Diagnóstico radiográfico y tratamiento quirúrgico de un caso clínico. Av. Odontoestomatol 2008; 24 (5): 307-312. 


\section{INTRODUCCIÓN}

El término "odontoma" fue introducido en 1867 por Broca. Es una neoplasia benigna mixta de origen odontógeno, es decir, es una lesión de células odontogénicas epiteliales y mesenquimatosas, completamente diferenciadas y que forman esmalte, dentina y cemento (1-4).

Los odontomas son los tumores odontogénicos con mayor frecuencia de aparición y representan el $51 \%$ de todos los tumores odontogénicos. Diversos autores han clasificado los odontomas de distintas formas. Así encontramos que los han dividido en odontoma ameloblástico, compuesto y complejo. El odontoma compuesto es una malformación en la que están representadas todos los tejidos dentarios con un patrón más ordenado que un odontoma complejo, de modo que la lesión consiste en muchas estructuras de aspecto dentario. La mayoría no mantiene la estructura de la dentición normal, pero en cada una el esmalte, la dentina, el cemento y la pulpa están dispuestos como en el diente normal. El odontoma complejo es una malformación en la que están representados todos los tejidos dentarios, en general bien formados individualmente pero dispuestos según un patrón más o menos desordenado. El odontoma ameloblásticos es una neoplasia, con las características generales del fibroma ameloblástico, pero que contiene dentina y esmalte en su estructura $(6,7,9,17)$.

El odontoma compuesto se presenta con frecuencia en la segunda década de la vida. Existe una ligera predilección por el sexo masculino $(8,9,17)$. Su localización es mayor en el maxilar superior, sector anterior, lado derecho. $(8,18,19)$ Es más frecuente el compuesto que el complejo (6).

Desde el punto de vista histopatológico el odontoma compuesto presenta pulpa, dentina primaria, esmalte hipocalcificado, cemento primario y un trabeculado óseo entre los dentículos. Existen formas mixtas con morfología intermedia $(10,11)$.

Radiográficamente el odontoma compuesto se presenta como una imagen mixta (radiopaca y radiolúcida), que adopta una configuración similar a dientes (dentículos), rodeados por un halo radiolúcido (7, 11, 12).
La mayor parte de los odontomas son asintomáticos, aunque pueden aparecer signos y síntomas relacionados con su presencia, tales como dientes supernumerarios, impactados, retenidos, inflamación e infección $(8,13,14)$.

El objetivo de este trabajo es estudiar al odontoma compuesto a través de la revisión de la literatura y presentación de un caso clínico de odontoma compuesto asociado a un diente supernumerario y un canino retenido, estudiado a través del diagnóstico radiográfico y su tratamiento quirúrgico.

\section{MATERIALES Y MÉTODOS}

Se realizaron estudios radiográficos panorámicos con un equipo ortopantomógrafo de movimiento helicoidal de última generación marca Cranex Thome con valores utilizados de $65 \mathrm{Kv}, 10 \mathrm{~mA}$; tiempo de exposición: 15 segundos.

Las películas utilizadas para esta técnica es de 15 cm por $30 \mathrm{~cm}$, marca Agfa y chasis rígido con pantallas reforzadoras sensible al espectro de luz verde.

Los estudios radiográficos intraorales se realizaron con un equipo radiográfico marca Dabi Atlante con valores fijos de $70 \mathrm{Kv}, 8 \mathrm{~mA}$ y tiempo de exposición promedio de $0,3 \mathrm{seg}$. por toma. Se utilizaron películas retroalveolares de $3 \mathrm{~cm}$ por $4 \mathrm{~cm}$ y oclusales de $6 \mathrm{~cm}$ por $8 \mathrm{~cm}$, marca Agfa. Para el procesado se utilizó una máquina automática marca AT/2000 (15).

Se utilizaron los elementos de protección según normas vigentes.

El tratamiento quirúrgico se llevó a cabo con material de antisepsia descartable, y para la confección de los campos. Anestesia local: 3 anestubos marca totalcaína, aguja de anestesia corta mango de bisturí $n^{\circ} 3$, hoja $n^{\circ} 15$; separadores de farabeuf, periostótomo, sindesmótomo, fresas quirúrgicas redondas $\mathrm{n}^{\circ} 4,6$ y 8, instrumental rotatorio con su fisiodispenser, pinzas Pean, Kocher, gubia. Cucharas y limas para hueso, hilo de sutura 0000, porta aguja, tijera, gasa estéril, solución fisiológica y solución desinfectante. 
Los estudios histopatológicos se realizaron en la Cátedra de Anatomía Patológica de la Facultad de Odontología de la Universidad de Buenos Aires.

\section{CASO CLÍNICO}

Concurre a la consulta un paciente de sexo masculino de 16 años, aludiendo dolor agudo y aumento de volumen en la zona canina derecha del maxilar superior. Se realiza un minucioso estudio clínico observándose la persistencia del canino temporario derecho, ausencia del 1.3 y un abombamiento de la zona de consistencia dura (Figura 1).

A partir de allí se realiza estudios radiográficos extraorales que consistentes en radiografía panorámica (Figura 2), estudios intraorales con técnica de Dieck (Figura 3) y técnica Oclusal lateralizada (Figura 4).

En la figura 2 se observa la presencia en el sector anterosuperior derecho una imagen mixta con múltiples radiopacidades, la persistencia del canino temporal la presencia del canino permanente en retención y por distal de su raíz la presencia de un diente supernumerario.

Se observa en la figura 3 una imagen radiográfica mixta, con múltiples radiopacidades que adoptan una configuración similar a dientes (dentículos), rodeados por un halo radiolúcido. Se identifica la presen-



Fig. 1. Imagen clínica.

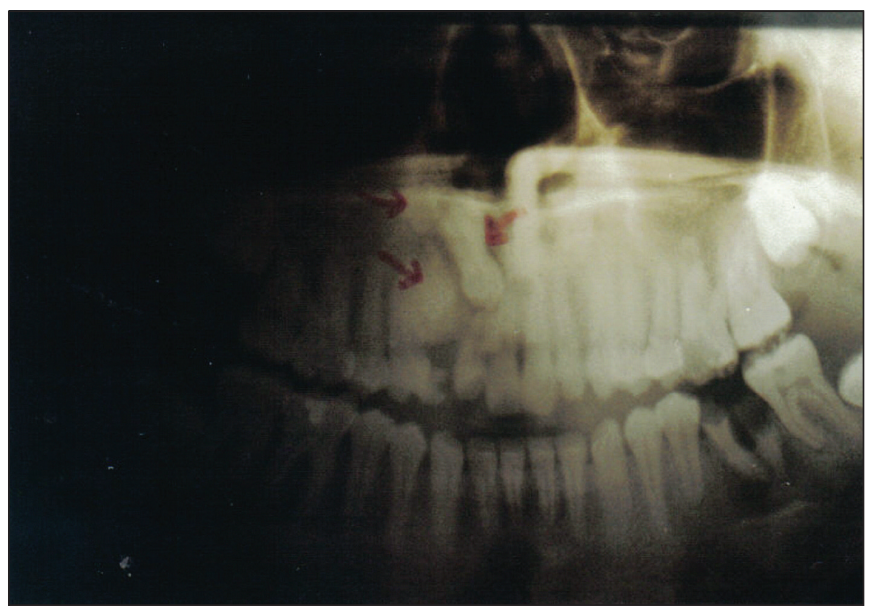

Fig. 2. Radiografía panorámica.

cia del canino temporal, el canino permanente retenido y un diente supernumerario.

Se observa en la figura 4 una estructura mixta con predominio radiopaco compatible con un odontoma, el diente supernumerario y el canino retenido.

Realizados todos lo estudios realizados se concluye en un diagnostico presuntivo de odontoma compuesto y se decide su remoción quirúrgica, la del canino temporario, el canino retenido y la pieza supernume-

Fig. 3. periapical.






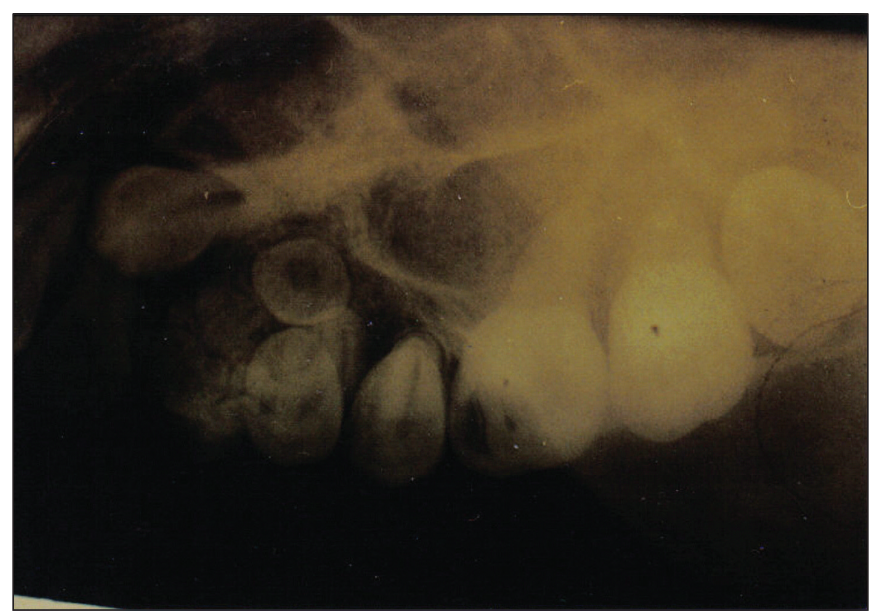

Fig. 4. Radiografía oclusal.

raria. Se presentan las fotos de lo distintos pasos quirúrgicos.

En la figura 5 se observa el colgajo elevado ya realizada la extracción de la pieza 5.3. La osteotomía permite visualizar la presencia de elementos compatibles con tejidos dentarios. En la figura 6 se realiza la eliminación de la tumoración en la que se visualiza con precisión la similitud con piezas dentarias en forma ordenada y de diferentes tamaños, lo que nos permite dar un diagnóstico intraquirúrgico de odontoma compuesto.

La figura 7 nos muestra la cavidad que dejó el odontoma, a la derecha la cavidad del supernu-

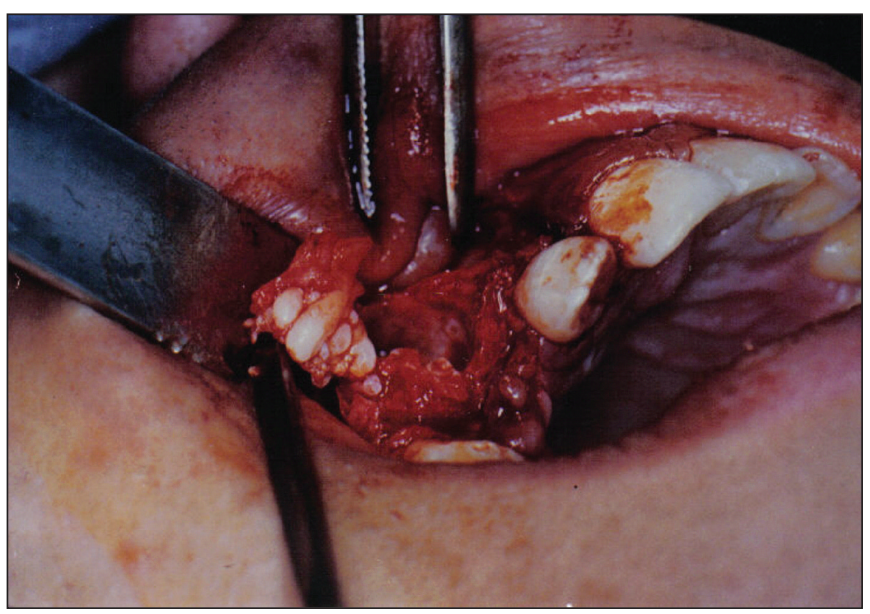

Fig. 6.

merario y el espacio que dejó el canino extraído. En la figura 8 se visualiza la reposición del colgajo y la sutura.

En la figura 9 se observa, en la parte inferior, el diente temporario, el supernumerario y el canino que fue seccionado para su exodoncia, por arriba se visualizan gran parte de los dentículos del odontoma. La figura 10 muestra la radiografía postoperatoria a distancia (12 meses posteriores a la cirugía) con una técnica periapical intraoral.

Examen histopatológico. Permitió establecer el diagnóstico definitivo de odontoma compuesto.

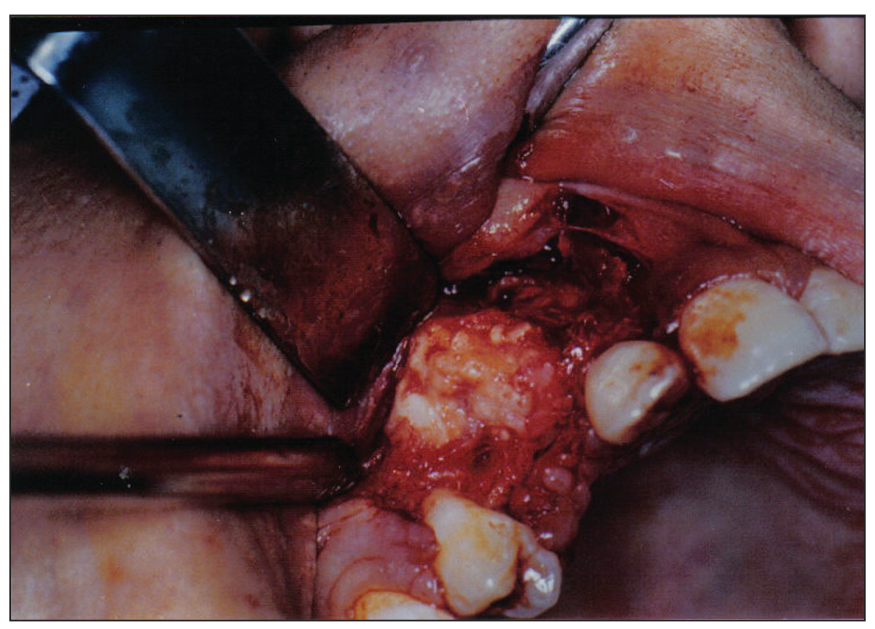

Fig. 5.

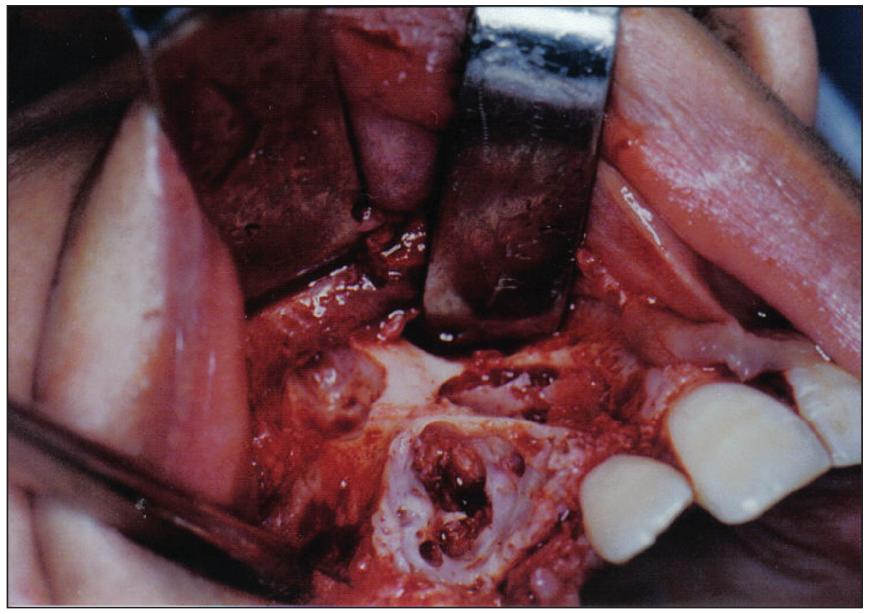

Fig. 7. 


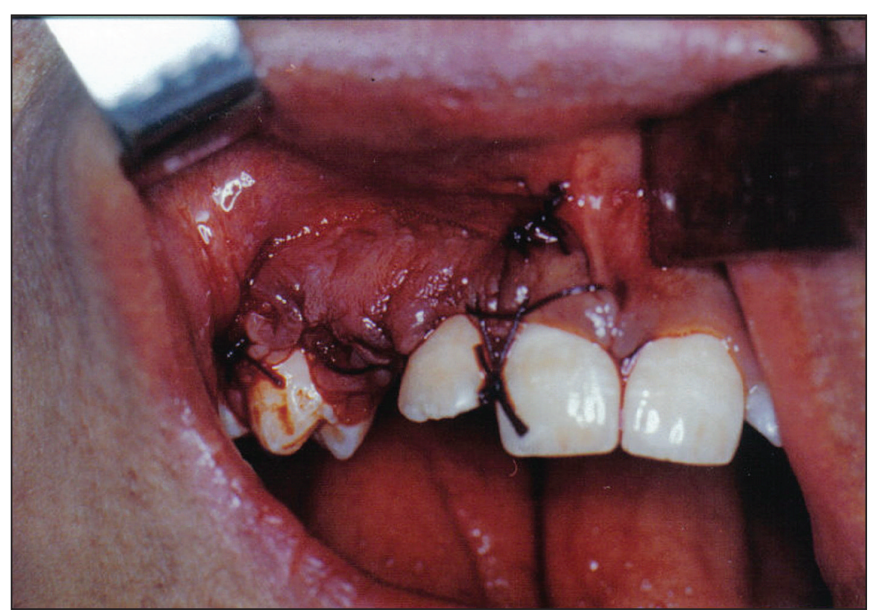

Fig. 8.

\section{DISCUSIÓN Y CONCLUSIÓN}

Se pudo establecer que la técnica panorámica es el estudio radiográfico aconsejable para complementar la clínica y contribuir al diagnóstico presuntivo ya que tiene como ventaja la posibilidad de observar una visión completa de ambas arcadas dentarias y sus estructuras vecinas (15). Las técnicas intraorales nos permitieron visualizar la lesión con mayor fidelidad dimensional y de detalle respecto a la anterior. La imagen radiográfica observada en nuestro caso clínico nos permitió ver una imagen mixta, con múltiples radiopacidades de configuración similar a piezas dentarias, presentado el conjunto un halo radiolúcido

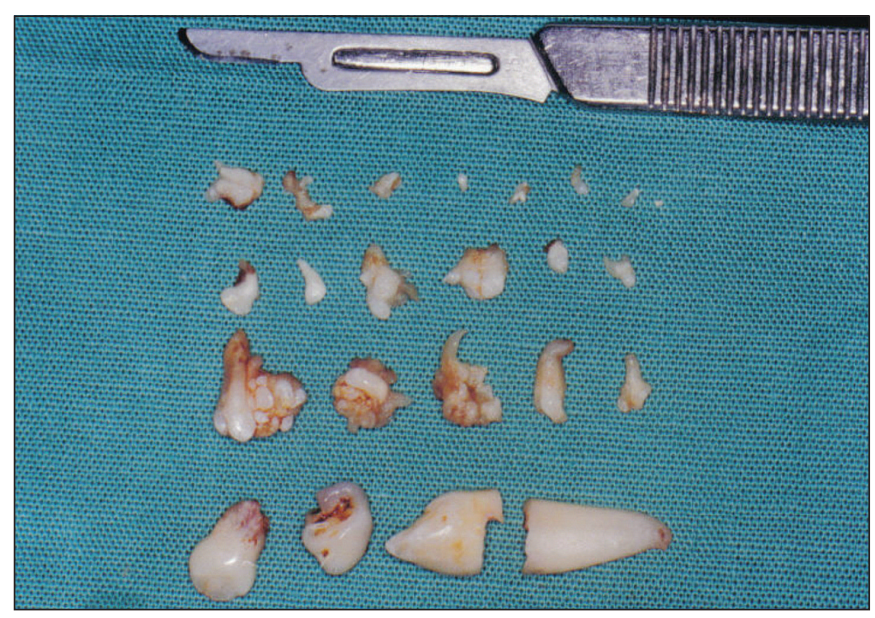

Fig. 9.

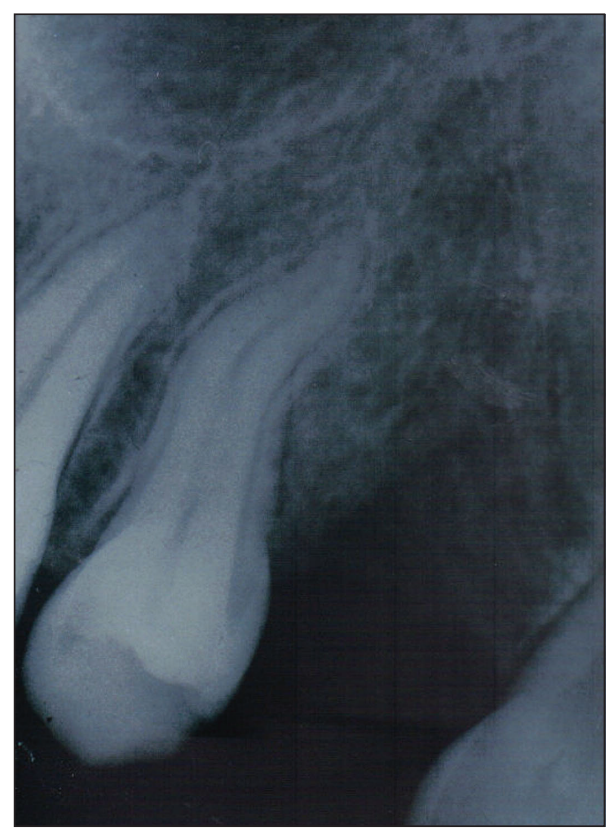

Fig. 10.

perimetral en coincidencia con las características que relatan los autores consultados (11-16).

El paciente tratado es del sexo masculino, en la segunda década de vida. La lesión se encontraba en el maxilar superior en la zona anterior del lado derecho coincidiendo con la prevalencia tanto en edad, sexo y ubicación del odontoma compuesto según la bibliografía detallada $(6,8,10,11,19,20)$.

Con este trabajo se demuestra la importancia de realizar un diagnóstico adecuado ante este tipo de afección en estadios tempranos. El análisis complejo, así como la evolución del caso, permitieron confirmar el diagnóstico inicial de odontoma compuesto. La corroboración a través de los estudios histopatológicos es imprescindible para el la resolución del caso y seguimiento. El tratamiento quirúrgico conservador dio un muy buen resultado en el mediano y largo plazo como se puede visualizar en los estudios radiográficos a distancia. Esto fue el resultado de un correcto diagnóstico clínico, radiográfico e histopatológico.

\section{BIBLIOGRAFÍA}

1. Lopez-Areal L, Silvestre Donat F, Gil Lozano J. Compound odontoma erupting in the mouth: 4- 
year follow up of a clinical case. J Oral Pathol Med. 1992;21(6):285.

2. Cataldo E, Santis HR. A clinico-pathologic presentation. Ameloblastic fibrous odontoma. J Mass Dent Soc. 1992;41(2):43.

3. Piattelli A, Trisi P. Ghost cells in compoun odontoma: a study of undemineralized material. Bull Groyp Int Rech Sci Stomatol Odontol. 1991;34 (3-4):145.

4. Owens BM; Schuman NJ; Mincer HH. Dental odontomas: a retrospective study of 104 cases. J Clin Pediatr Dent. 1997;21(3):261.

5. Delgado WA, Arrascue M, Calderón V, Paniura D. Tumor Odontogénico Híbrido: tumor odontogénico quístico calcificante con odontoma complejo y focos de ameloblastoma Rev. Estomatol. Herediana 2006;16(2).

6. Dinatale E. Neuralgia sintomática de la tercera rama del trigémino asociada a un odontoma compuesto. Presentación de un caso. Acta Odontol Venez 2003;41(3):50-3.

7. Ferrer MJ, Silvestre FJ, Estrelles E, Grau D, López R. Recurrent infection of a complex odontoma following eruption in the mouth. Med Oral 2001;6:269-5.

8. Ragalli CC, Ferreria JL, Blasco L. Large erupting complex odontoma. Int J Oral Maxillofac Surg 2000;29:373-4.

9. Amado S, Gargallo J, Berini L, Gay C. Revisión de 61 casos de odontoma. Presentación de un odontoma complejo erupcionado. Med Oral 2003;8:366-73.

10. Hisatomi M, Asaumi JI, Konouchi H, Honda Y, Wakasa T, Kishi K. A case of complex odontoma associated with an impacted lower deciduous second molar and analysis of the 107 odontomas. Oral Dis 2002;8:100-5.

11. De Oliveira BH, Campos V, Marcol S. Compound Odontoma-diagnosis and treatment: Three cases report. Pediatr Dent 2001;23(2):151-7.
12. Nelson P, Silva A, Farias G, Freitas AC. Odontoma-hice malformation on in a permanent maxillary central incisor subsegment to trauma to the incisor predecesor. Dent Traumatol 2005; 21(5):309-12.

13. Bantra P, Dugal R, Kharbanda OP, Porkous H. Odontogenic treatment of impacted anterior teeth due to odontomas a report of two cases. J Clin Pediatric Dental 2004;28(4):289-94.

14. De Alesio D, Creosinin ML. Odontoma compuesto complejo: un caso clinico. Rev Asoc Odontol Argent 2003;91(5):433-6.

15. Rushton VE, Horner K. The use of panoramic radiology in dental practice. J Dent. 1996;24:185201.

16. Amado Cuesta S, Gargallo Arbiol J, Berini Ayt s L, Gay Escoda C. Ti: Revisión de 61 casos de odontoma complejo eupcionado compuesto: reporte de un caso. Fo: Med. oral. 2003;8(nov-dic 5):366-73.

17. Duvegneaud S, Tant L, Loeb I, Kaumpuridis S, Dargent JL, Gomez Galdon M, Vilbi M,Van Reck J. Ti: Mandibular Ameloblastic fibro-odontoma: case report. Fo: Rev. Stomatol Chir Maxillofac. 2004 Sep; 105(4):223-6.

18. Mupparapu M, Singer SR, Rinaggio J. Ti: an unusualliy large complex odontoma of the maxilla. Fo: Quintessence Int. 2004;35(sep 8): 641-5.

19. Ikésima A,Tamura Y. Differrential diagnosis between dentigerous cyst and benign tumor with and embedded tooth. J Oral Sci 2002;44(1):13-7.

20. Tomizawa M, Otsuka Y, Noda T. Ti: Clinical observations of odontomas children: 39 cases including one recurrent case. Fo: Int. J Paedia Dent. 2005;15(Jan 1):37-43.

\section{CORRESPONDENCIA}

Gdor. Marcelino Ugarte $15481^{\circ}$ piso depto. "C" Olivos C P 1636

Provincia de Buenos Aires. Argentina

E- Mail: jv983@hotmail.com.ar

eecarbajal@yahoo.com.ar 